\title{
An NMR Study on the Phase Change of Lipid Membranes by an Antimicrobial Peptide, Protegrin-1
}

\author{
Chul Kim \\ Department of Chemistry, Hannam University, Daejeon 305-811, Korea.E-mail: chulkim@hnu.kr \\ Received November 4, 2009, Accepted December 23, 2009
}

\begin{abstract}
Membrane disruption by an antimicrobial peptide, protegrin-1 (PG-1), was investigated by measuring the ${ }^{2} \mathrm{H}$ solid-state nuclear magnetic resonance (SSNMR) spectra of 1-palmitoyl- $d_{31}$-2-oleoyl-sn-glycero-3-phosphatidylcholine (POPC $\left.d_{31}\right)$ in the mixture of PG-1 and POPC_ $d_{31}$ lipids deposited on thin cover-glass plates. The experimental line shapes of anisotropic ${ }^{2} \mathrm{H}$ SSNMR spectra measured at various peptide-to-lipid $(\mathrm{P} / \mathrm{L})$ ratios were simulated reasonably by assuming the mosaic spread of bilayers containing pore structures or the coexistence of the mosaic spread of bilayers and a fast-tumbling isotropic phase. Within a few days of incubation in the hydration chamber, the pores were formed by the peptide in the POPC $d_{31}$ and POPC_ $d_{31} /$ cholesterol membranes. However, the formation of the pores was not clear in the POPC_d $d_{31} / 1$-palmitoyl-2-oleoyl-sn-glycero-3-phosphatidylglycerol (POPG) membrane. Over a hundred days after hydration, a rapidly rotating isotropic phase increased in the POPC $d_{31}$ and the POPC $d_{31} /$ cholesterol membranes with the higher $\mathrm{P} / \mathrm{L}$ ratios, but no isotropic phase appeared in the POPC $\bar{d}_{31} / \mathrm{POPG}$ membrane. Cholesterol added in the POPC bilayer acted as a stabilizer of the pore structure and suppressed the formation of a fast-tumbling isotropic phase.
\end{abstract}

Key Words: Antimicrobial peptide, Protegrin-1, Toroidal pore, ${ }^{2} \mathrm{H}$ solid-state NMR, Phase change

\section{Introduction}

Antimicrobial peptides (AMPs) are one of the innate immune systems in mammals, amphibians, and insects and are used to kill a broad range of pathogens such as viruses, bacteria, and fungi. ${ }^{1-6}$ Their broad activities are strongly related to the fact that except for a small group of AMPs that act via a receptormediated disruption mechanism, most AMPs act on membranes via a nonreceptor-mediated disruption mechanism. ${ }^{7}$ The AMP interaction with a membrane depends on the membrane properties, such as a surface charge density, ${ }^{8}$ membrane rigidity at a hydration level, ${ }^{9}$ and the AMP properties, such as a peptide conformation at the membrane-binding state ${ }^{10}$ and peptide charge density. ${ }^{11}$ However, the common characteristics of the peptide-lipid interaction are 2 interactions of an electrostatic interaction between highly charged residues of the peptide and a surface charge in the polar head group of the lipid, and a hydrophobic interaction between the hydrophobic side of the peptide and the alkyl chain of the lipid. ${ }^{12}$

While AMPs kill microbes by disrupting the cell membranes, the detailed mechanisms for the membrane disruption are diverse for the different types of AMPs. ${ }^{13}$ The most plausible mechanisms proposed for the membrane disruption include a formation of micelles, a carpet model, a membrane thinning model, a barrel-stave model, and a toroidal pore model. ${ }^{13-15}$ When peptides bind onto the lipid membrane, the binding site in the membrane and the conformation of the peptide depend, in general, on the peptide concentration, surface charge density, and membrane ingredients such as cholesterol. For example, some AMPs have 2 states depending on the peptide concentration. At a low peptide-to-lipid (P/L) ratio, the peptide is in the "S" state in which the peptide binds on the membrane surface with a $\beta$-sheet orientation parallel to the plane of the bilayers, and above a threshold value, the peptide is in the "I" state in which the peptide inserts into the bilayer and is in the orientation perpendicular to the plane of the bilayers. ${ }^{16}$ Rhesus theta defensin-1 (RTD-1), a $\theta$-defensin discovered in granulocytes of the rhesus monkey, ${ }^{17}$ causes the formation of lipid cylinders in the POPC/POPG membranes, whereas protegrin-1 (PG-1) breaks the POPC/POPG membrane into small vesicles. ${ }^{18}$ Alamethicin, a 20 -amino acid peptide that has been isolated from the fungus Trichoderma viride, ${ }^{19}$ and magainin, an $\alpha$-helical structured 23residue peptide found on the skin of the African clawed frog Xenopus laevis, ${ }^{20-21}$ only thinned the membrane without disruption of the bilayer at specific $\mathrm{P} / \mathrm{L}$ ratios. ${ }^{22}$ A short polycationic HIV-derived cell-penetrating peptide TAT induces presumably inverted micelles assembled into bundles in the zwitterionic dimyristoylphosphatidylcholine (DMPC), but not in the anionic dimyristoylphosphatidylglycerol (DMPG) bilayers. ${ }^{23}$ Pandinin 2, a pore-forming peptide isolated from the crude venom of the African scorpion Pandinus imperator, ${ }^{24}$ lyses membranes only under acidic conditions and at temperatures below the liquid crystal-gel phase transition of the lipid bilayers after incubation for 2 days in the magnet. ${ }^{25}$ Gramicidin S appears to be located in a more polar environment of the bilayer surface when cholesterol is present because of the attenuating effect of cholesterol on the interactions of Gramicidin $\mathrm{S}$ with phospholipids bilayers. ${ }^{26}$ As this brief survey shows, there are many different mechanisms involved in as many cases. Therefore, more investigations will be required for the deeper understanding of antimicrobial actions.

Protegrin-1 (MW = 2154 Da) is an 18-residue AMP found in porcine leukocytes. ${ }^{27}$ It shows a broad range of antimicrobial activity in that it can kill Gram-negative bacteria, Gram-positive bacteria, and fungi and also displays modest antiviral activity against the HIV-1 virus. PG-1, which has 2 disulfide bonds among the 4 Cys residues, forms an antiparallel $\beta$-strand in solution (length: $30 \AA$, cross section: $\sim 8 \AA$ ). ${ }^{28}$ Studies carried out by 
a neuron diffraction, nuclear magnetic resonance (NMR), etc., have reported that PG-1 causes largely static disorder in the various lipid membranes by forming a pore structure or a local thinned structure, which depends on the lipid types, addition of anionic lipids, and peptide concentrations. ${ }^{8,29-34}$ The total interaction of PG-1 with POPC is the outcome of both the electrostatic and van der Waals' interactions, whereas the total interaction of PG-1 with POPC/POPG derives dominantly from the contribution of the electrostatic interaction. The local thinning of the lipid bilayer mediated by the PG-1 monomer is enhanced in the POPC/POPG membrane. ${ }^{35}$ The bound structure and the dimerization of PG-1 on the membrane surface have been also investigated using various spectroscopic techniques. ${ }^{36-38}$

Solid-state NMR spectroscopy is widely used to study the structure of small insoluble peptides by measuring multinuclear spectra. ${ }^{8,33,34,39}$ In particular, ${ }^{31} \mathrm{P}$ and quadrupolar ${ }^{2} \mathrm{H}$ SSNMR spectra of peptide-lipid supramolecular assemblies provide the orientational distribution of the membrane lipids in the presence of AMPs and dynamic characters of lipids. ${ }^{40}$ The simulated SSNMR spectra, using an appropriate geometric model for the membrane disruption, have given abundant information on the disrupted feature of the lipid-peptide assemblies. ${ }^{8,34}$

To understand the membrane disruption by PG- $1,{ }^{2} \mathrm{H}$ SSNMR experiments were performed with the mixture of PG-1 and POPC $d_{31}$ lipids deposited on thin cover-glass plates. The time change of the ${ }^{2} \mathrm{H}$ SSNMR spectra was measured in the presence of PG- 1 at the P/L ratios of $1 / 80,1 / 50$, and $1 / 20$. The effects of an anionic lipid, POPG, and cholesterol on membrane bilayer were also investigated.

\section{Experimental}

Materials. 1-Palmitoyl- $d_{31}$-2-oleoyl-sn-glycero-3-phosphatidylcholine (POPC_ $d_{31}$ ) and 1-palmitoyl-2-oleoyl-sn-glycero3-phosphatidylglycerol (POPG) were purchased from Avanti Polar Lipids, Inc. (Alabaster, AL, USA). PG-1 (RGGRLCYCRRRFCVCVGR) was purchased from GL Biochem (Shanghi, China) and used without any further purification. Trichloroethanol (TFE), chloroform, cholesterol, and sodium phosphate dibasic were purchased from Aldrich Chemicals (Milwaukee, WI, USA). Thin cover-glass plates of $\sim 80 \mu \mathrm{m}$ thickness cut into rectangles of $10 \times 10 \mathrm{~mm}$ in width were purchased from Marienfeld Laboratory Glassware (Bad Mergentheim, Germany).

NMR sample preparation. Mechanically aligned lipid membranes were prepared on thin cover-glass plates to produce a multi-bilayered membrane mimic system by following the standard procedure developed by Hallock et al. ${ }^{41}$ PG-1 was dissolved in TFE and mixed with chloroform solutions containing the appropriate lipids. The $\mathrm{P} / \mathrm{L}$ molar ratios were adjusted as $1 / 80$, $1 / 50$, and $1 / 20$. For the POPG-containing membrane, the mixture of POPC $d_{31}$ and POPG with a molar ratio of 3:1 was used. The mixture of POPC_ $d_{31}$ and cholesterol with a molar ratio of 1:1 was used for the cholesterol-containing membrane. The homogeneously mixed solutions were deposited onto each coverglass plate at a surface concentration of $0.01-0.04 \mathrm{mg} / \mathrm{mm}^{2}$, dried for 4 hours in air, and then vacuum-dried overnight to remove residual organic solvents thoroughly. The dried samples were directly hydrated by dropping $2 \mu \mathrm{L}$ of water ${ }^{29,39}$ and placed for 2 days in a chamber containing a saturated sodium phosphate dibasic solution, which provides $95 \%$ relative humidity. About 10 or 15 thin cover-glass plates with the hydrated sample were stacked, wrapped in Parafilm, and sealed in a polyethylene bag to prevent them from drying during the NMR measurements. After a few days of incubation in a refrigerator at $5{ }^{\circ} \mathrm{C}$ for the stabilization of the sample, the samples were inserted into a rectangular NMR probe coil and their static ${ }^{2} \mathrm{H}$ SSNMR spectra were measured. The samples after the first NMR measurement were stored in the refrigerator at $5^{\circ} \mathrm{C}$ for hundreds of days until the next NMR measurements.

Solid-state ${ }^{2} \mathbf{H}$ nuclear magnetic resonance spectroscopy. The SSNMR experiments were carried out on a Bruker Avance II $300 \mathrm{MHz}$ spectrometer operating at the resonance frequencies of $300.12 \mathrm{MHz}$ for ${ }^{1} \mathrm{H}$ and at $46.07 \mathrm{MHz}$ for ${ }^{2} \mathrm{H}$. A static $\mathrm{H}-\mathrm{X}$ double resonance probe with a flat rectangular coil with the dimensions of $18 \times 10 \times 5 \mathrm{~mm}$ was used for measuring the static ${ }^{2} \mathrm{H}$ NMR spectrum. ${ }^{2} \mathrm{H}$ SSNMR spectra were acquired using a quadrupolar echo sequence with an echo delay time of $30 \mu$ s and a recycle delay of $0.5 \mathrm{~s}$. The typical ${ }^{2} \mathrm{H} 90^{\circ}$ pulse length was 5.0 $\mu \mathrm{s}$. The spectral width for ${ }^{2} \mathrm{H}$ spectra was $100 \mathrm{kHz} .{ }^{2} \mathrm{H}$ spectra were typically averaged over 12,000 scans. All experiments were carried out at $20{ }^{\circ} \mathrm{C}$.

\section{Theoretical Considerations}

The ${ }^{2} \mathrm{H}$ SSNMR spectrum is an ideal tool for investigating the distribution of lipids forming biomembranes. ${ }^{42,43}$ The anisotropic SSNMR line shape of ${ }^{2} \mathrm{H}$ quadrupolar coupling interaction depends on the orientation of anisotropic quadrupolar coupling tensor of ${ }^{2} \mathrm{H}$ spin in the lipid molecule with respect to the external magnetic field $\mathrm{B}_{0}$. In our sample, which was mechanically aligned on the thin cover-glass plate, the orientation of lipid molecule was determined by both the orientation of the glass plate normal direction with respect to $\mathrm{B}_{0}$ field and the relative orientation (angular distribution) of the motionally averaged principal axis of lipid molecule with respect to the glass plate normal.

To simulate the observed ${ }^{2} \mathrm{H}$ SSNMR line shapes of POPC $d_{31}$ lipids distributed on the curved membrane surfaces, the SSNMR line shape factors were calculated by using simple algebraic calculations. The SSNMR line shape factor is directly proportional to infinitesimal surface area at a certain orientation angle $(\Omega)$ of the molecular axis frame of the lipid POPC_ $d_{31}$ with respect to the glass plate normal and also gives the signal intensity of ${ }^{2} \mathrm{H}$ SSNMR spectrum at the anisotropic SSNMR frequency when the lipid molecule is at the orientation angle, $\Omega$. The overall SSNMR line shape was determined by integrating the intensity values over the whole range of the SSNMR frequencies. The detailed processes determining the SSNMR line shape factors and the lateral diffusion effects for the pore distribution and the thinned distribution in the thinned membrane bilayer were reported in the previous paper. ${ }^{34}$

The POPC_ $d_{31}$ lipid molecule used in our experiment has 15 types of deuterons in its aliphatic part and, therefore, shows the spectrum summed over $15^{2} \mathrm{H}$ SSNMR anisotropic line shapes, where each ${ }^{2} \mathrm{H}$ spectral line shape depends on the angular distribution of lipids on the thin glass plate. Fig. 1 shows the 
$\mathrm{B}_{0}$

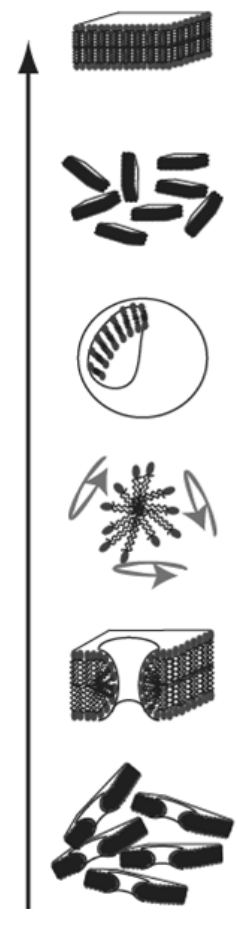

(A)

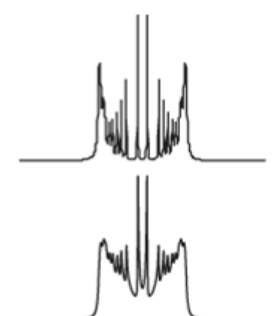

(C)

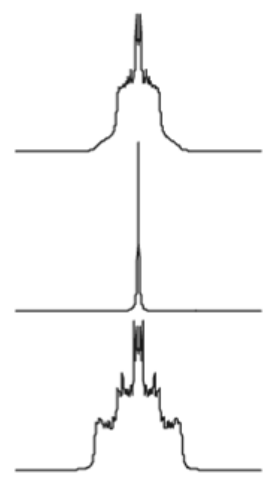

(F)

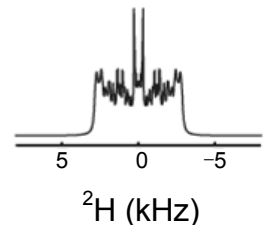

Figure 1. Simulated ${ }^{2} \mathrm{H}$ SSNMR spectra of POPC $d_{31}$ in various lipid distributions. (A) Well-aligned bilayer, (B) mosaic spread of bilayers by a Gaussian function with the standard deviation of $25^{\circ}$, (C) liposome, (D) randomly fast rotating micelle, (E) toroidal pore, and (F) Gaussian spread of bilayers containing toroidal pores with the standard deviation of $25^{\circ}$.

simulated ${ }^{2} \mathrm{H}$ SSNMR spectra that can be measured in some typical distributions of POPC $d_{31}$ molecules. Here, the 15 quadrupolar coupling constants (QCC) values (data not shown) were used, which fit the experimental ${ }^{2} \mathrm{H}$ SSNMR spectrum to the best of simulation. ${ }^{8,34}$ When only the lipids were deposited onto the thin cover-glass plates and well hydrated in the chamber, the lipids were well aligned to the direction of glass plate normal. Then, the ${ }^{2} \mathrm{H}$ SSNMR spectrum with 30 sharp peaks as shown in Fig. 1A will be obtained. However, when the lipid bilayer normal axes are not well aligned and are spread by the mosaic distribution, ${ }^{44}$ then the ${ }^{2} \mathrm{H}$ SSNMR spectrum will be like Fig. 1B when simulated with a Gaussian angular distribution function having the standard deviation of $25^{\circ}$. As the standard deviation of a Gaussian distribution increases, the central portion of the ${ }^{2} \mathrm{H}$ spectrum that has a higher intensity converges into the line shape that is similar but not the same as that of the spectrum of the randomly distributed lipids, as shown in Fig. 1C. For the spherical distribution of lipids, such as a liposome shown in Fig. $1 \mathrm{C}$, the ${ }^{2} \mathrm{H}$ SSNMR spectrum shows the characteristic line shape that has higher intensity around the center of the spectrum and small shoulders at the edge. However, if the lipid aggregates such as liposomes or micelles rotate randomly with a fast rotation frequency, the ${ }^{2} \mathrm{H}$ SSNMR line shape will collapse into a single sharp peak at $0 \mathrm{kHz}$ as shown in Fig. 1D.

Some AMPs, including PG-1, have been known to make

pores in the bilayer membranes made out of POPC lipids. Ramamoorthy et al. proposed the toroidal pore with a circular shape on the membrane surface. ${ }^{41}$ In this case, the ${ }^{2} \mathrm{H}$ NMR spectrum of POPC_ $d_{31}$ will be simulated as shown in Fig. 1E. Like bilayers spreading their orientation by a Gaussian angle distribution with the various standard deviations, the bilayers with pores also may spread with a larger standard deviation. For one of these cases, the ${ }^{2} \mathrm{H}$ SSNMR spectrum is given in Fig 1F. Here, all the simulated spectra were obtained by using the Matlab program coded by the author. From these features, one can easily confirm the formation of toroidal pores and the Gaussian spreads of the bilayers containing the pore structures in the presence of PG-1.

\section{Results and Discussion}

Phase changes of POPC bilayers. POPC, having a choline group as the head group and 2 aliphatic hydrocarbons as the tail group, is one of the main lipids constituting biomembranes. When PG-1 acts on the POPC bilayer, a pore structure is well known to be induced by PG-1 dimers. ${ }^{36}$ The induced pore structure was investigated by analyzing the ${ }^{31} \mathrm{P}$ and ${ }^{2} \mathrm{H}$ SSNMR spectra of the mixture of peptides and lipids deposited on a thin glassplate. In a general procedure, the lipid bilayer distorted by AMPs is hydrated by being placed in a refrigerator for 2 or 3 days before taking NMR measurements. 2 or 3 days of incubation is considered to be sufficient time for the lipid-peptide mixture to attain an equilibrated state, which may be a well-aligned lipid bilayer containing the pores. However, a few days of incubation was not found to be enough, as shown in Fig. 2. The spectrum (Fig. 2B) with a $\mathrm{P} / \mathrm{L}$ ratio of $1 / 80$ at 100 days later after hydrating shows similar spectrum as Fig. 1A, which means relatively wellaligned bilayer state. However, the spectrum (Fig. 2A) taken within a few days ( 7 days) shows significant distortion of the aligned bilayers.

This distortion was not among the mosaic spread of bilayers, isotropic phase, and toroidal pore, such as those in the simulated spectra seen in Fig. 1. The spectra cannot be fitted well only with a Gaussian spread of bilayers having the wide ranges of standard deviation, which was previously reported. ${ }^{8}$ The distorted line shape in Fig. 2A resembles the spectrum of Fig. 1F to a great extent, which can be obtained from a Gaussian distribution of lipid bilayers containing the toroidal pores; the line shape in Fig. 2B resembles the spectrum of Fig. 1A. First, the spectrum of Fig. 2D was simulated by assuming that some portions of the total lipids participate in the formation of pores in the lipid bilayer and the bilayer are well aligned. The best spectrum (Fig. 2D) was simulated by adjusting the portion of the total lipids participating in the pore structures. Second, the experimental spectrum (Fig. 2A) at the initial state was simulated as shown in Fig. $2 \mathrm{C}$ with the same portion of pore lipids as used in the simulation of the spectrum of Fig. 2D while adjusting the standard deviation of a Gaussian distribution of the bilayers. The simulated spectra of the sample with the $\mathrm{P} / \mathrm{L}$ ratio of $1 / 80$ at 100 days after hydrating the sample resulted in $30 \%$ of the total lipids being in the pore structures and $70 \%$ of the total lipids being in the bilayer structure. When the spectrum was simulated to fit the experimental spectrum (Fig. 2A) at an initial state of the sample with $30 \%$ of the pore lipids, a standard deviation of 


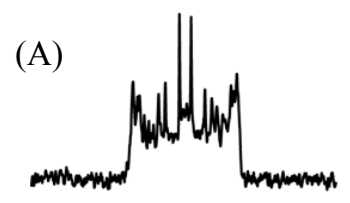

(B)

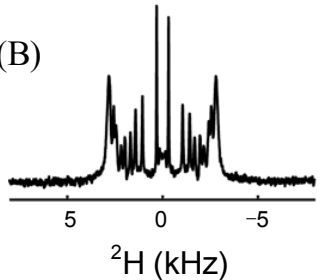

(C)

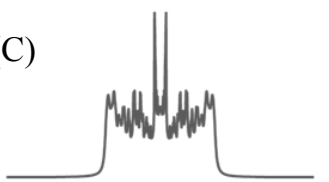

(D)

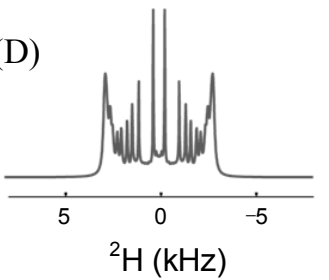

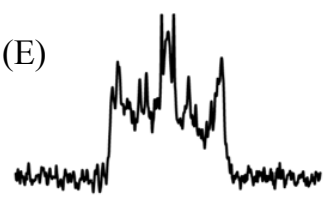

(F)

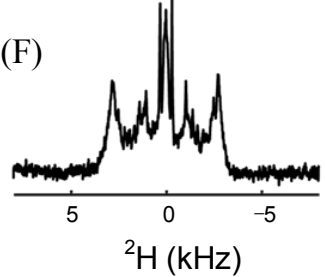

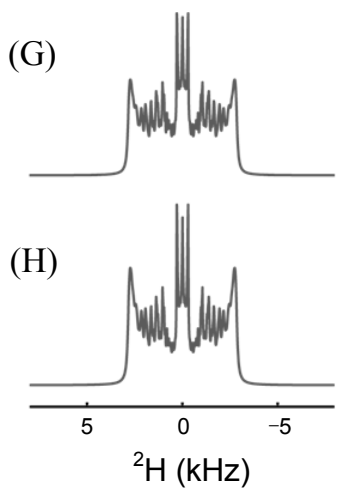

Figure 2. Experimental (A and $\mathrm{B}, \mathrm{E}$ and $\mathrm{F})$ and simulated $(\mathrm{C} \text { and } \mathrm{D}, \mathrm{G} \text { and } \mathrm{H})^{2} \mathrm{H}$ SSNMR spectra of POPC $d_{31}$ in the POPC membrane distorted by PG-1. P/L ratios are 1/80 (A-D) and 1/50 (E-H). Spectra of A and E were measured within a few days after sampling, and spectra of B and F were measured 100 days later.

$25^{\mathrm{O}}$ was obtained for the best simulation (Fig. 2C), which means that the mixture of peptides and lipids did not reach the equilibrated phase within a few days of incubation at $5^{\circ} \mathrm{C}$ in a refrigerator when the sample was prepared by this procedure. In contrast to the pure lipid bilayers, when the pores were formed by PG-1 in the bilayers, the bilayers may be more distorted off the well-aligned phase and will align more much slowly to the direction of the glass plate surface normal.

Some change was also found in the ${ }^{2} \mathrm{H}$ SSNMR spectra (Fig. $2 \mathrm{E}$ and $\mathrm{F}$ ) of the sample with the $\mathrm{P} / \mathrm{L}$ ratio of $1 / 50$. At first measurement, the ${ }^{2} \mathrm{H}$ NMR spectrum (Fig. 2E) showed that $30 \%$ of the total lipids participate in the formation of pores same as $1 / 80$ sample and the standard deviation of a Gaussian spread is $25^{\circ}$. However, the QCC values changed a little from those of the $1 / 80$ sample and had an additional isotropic phase of about $5 \%$ of which the peptide-lipid assembly tumbled very fast. In this $\mathrm{P} / \mathrm{L}$ ratio, the lipid bilayers were not well aligned even after 100 days unlike the spectrum in Fig. 1B.

At the initial states of the 2 samples with the $\mathrm{P} / \mathrm{L}$ ratios of $1 / 80$ and $1 / 50$, there was no significant difference in both the portion of pore structures and the standard deviations of Gaussian spreads, except for a small portion of isotropic phase in the $1 / 50$ sample. However, the ${ }^{2} \mathrm{H}$ SSNMR spectra of the $1 / 20$ sample showed dramatic changes in the initial and the final states, as shown in Fig. 3. At the initial state, the spectrum (Fig. $3 \mathrm{~A}$ ) of the $1 / 20$ sample showed a feature of the mixture of an isotropic phase and a Gaussian spread of bilayers containing pores with large standard deviations. The best simulation (Fig. 2D) for the experimental spectrum was when $10 \%$ of the lipids was in an isotropic phase, $30 \%$ in the pore structures, and $60 \%$ in the bilayers. The standard deviation of $90^{\circ}$ in a Gaussian spread function was used for simulating the experimental spectrum. The isotropic phase of $10 \%$, which is higher than $5 \%$ of the $1 / 50$ sample, is considered to be the reason for the significant mosaic spread of the bilayers. It is likely that the isotropic phase in the middle of the bilayers inhibits the alignment of bilayers because of its spherical shape, which is also the probable reason of the bad alignment of bilayers in the $1 / 50$ sample even 100 days later. As the time length increases, the isotropic phase increases and shows a sharper ${ }^{2} \mathrm{H}$ NMR peak at $0 \mathrm{ppm}$, which means that the rotational motion of the lipids in the isotropic phase is more

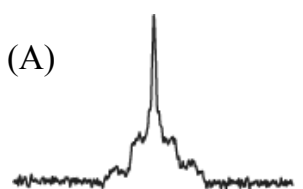

(D)
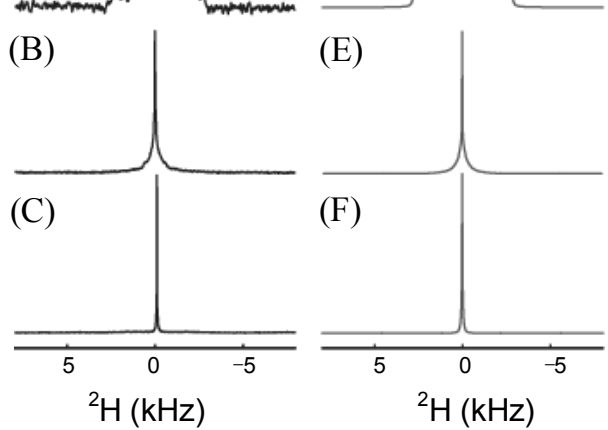

Figure 3. Experimental and simulated ${ }^{2} \mathrm{H}$ SSNMR spectra of POPC $d_{31}$ membrane distorted by PG-1 with the $\mathrm{P} / \mathrm{L}$ ratio of $1 / 20$. (A-C) Experiment and (D-F) simulation. Spectra were measured (A) within a few days after sampling, (B) 100 days after sampling, and (C) 200 days after sampling. The spectra were simulated with (D) $10 \%$ of the isotropic phase and $30 \%$ of the pore phase with a standard deviation of $90^{\circ}$, (E) $100 \%$ of the isotropic phase and its lateral diffusion coefficient of $D_{\mathrm{ld}}=10^{-12} \mathrm{~m}^{2} / \mathrm{s}$ and $(\mathrm{F}) 100 \%$ of an isotropic phase and its lateral diffusion coefficient of $D_{\text {ld }}=10^{-8} \mathrm{~m}^{2} / \mathrm{s}$.

rapid and therefore translates to a smaller size of a peptide-lipid assembly. Finally, all the lipids resulted in an isotropic phase after a long time of 100 days. By assuming the peptide-lipid assembly as a micelle with a diameter of $40 \AA$, the lateral diffusion coefficients of $D_{\mathrm{ld}}=10^{-12} \mathrm{~m}^{2} / \mathrm{s}$ and $D_{\mathrm{ld}}=10^{-8} \mathrm{~m}^{2} / \mathrm{s}$ were obtained.

Phase changes of POPC/POPG bilayers. To understand the effect of an anionic lipid, a POPC/POPG lipid system with the ratio of 3:1 was prepared with the same procedure as that used in the POPC bilayers. The ${ }^{2} \mathrm{H}$ SSNMR spectra were measured within a few days and at 100 days after starting the sample hydration. The NMR spectra in Fig. 4 show little effect of the peptide concentration on the membrane distortion. Unlike the POPC bilayer, when the experimental spectra (Figs. 4A, 4E, and 4I) were simulated with the mixture model composed of an isotropic phase and the mosaic spread of bilayers, the experimental 

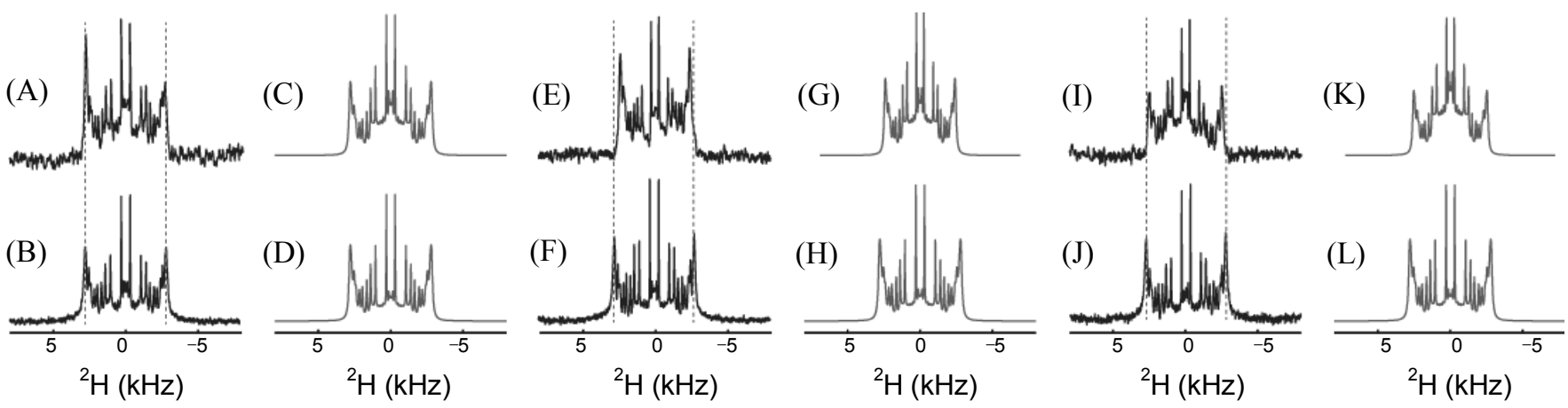

Figure 4. Experimental (A, B, E, F, I, and J) and simulated (C, D, G, H, K, and L) ${ }^{2} \mathrm{H}$ SSNMR spectra of the POPC $d_{31}$ in POPC $d_{31} / \mathrm{POPG}$ membrane disturbed by PG-1. P/L ratios are 1/80 (A-D), 1/50 (E-H), and 1/20 (I-L). Spectra in the top were measured within a few days after sampling and those in the bottom were measured 100 days later. Simulated spectra were obtained by summing 2 spectra of the aligned bilayers and an isotropic phase. The percentages of lipids involved in the isotropic phase are $50 \%(\mathrm{C}$ and $\mathrm{G}), 60 \%(\mathrm{~K})$, and $30 \%(\mathrm{D}, \mathrm{H}$, and $\mathrm{L})$. Dashed lines are given for eye guides of the anisotropic span of the spectrum.
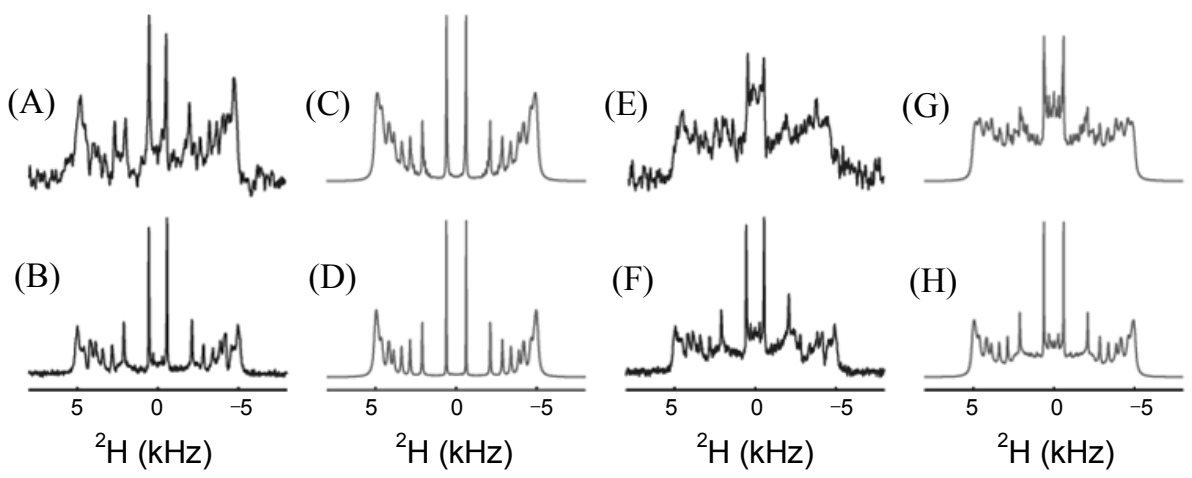

Figure 5. Experimental (first and third columns) and simulated (second and fourth columns) ${ }^{2} \mathrm{H}$ SSNMR spectra of POPC $d_{31}$ in POPC $d_{31} /$ cholesterol membrane disturbed by PG-1. P/L ratios are 1/80 (A-D), and 1/50 (E-H). Spectra in the top were measured within a few days after sampling and those in the bottom were measured 100 days later.

spectra were well fitted rather than the mosaic spread model of pore-containing bilayers used in POPC bilayer case. In the mosaic spread of the bilayers, the sharp peaks at the edge in the spectrum are smoothed as shown in the simulated spectra of Fig. 2C. The experimental spectra of Fig. 4 show sharp peaks, which means the mosaic spread is not significant and that the bilayers in the samples are relatively well aligned. From the simulated spectra (Figs. 4D, $4 \mathrm{H}$, and $4 \mathrm{~L}$ ), $30 \%$ of the total lipids were found to be involved in the isotropic phase at equilibrated states and about $50-60 \%$ at the initial states.

PG-1, having the positively charged Arg residues at the two ends of the long axis of the $\beta$-sheet, will be expected to bind more tightly on an anionic lipid-containing membrane and distort the bilayer more effectively. Mei Hong et al. reported that while PG-1 causes a largely static disorder in the POPC membrane, PG-1 breaks the membrane into small vesicles by the formation of a peptide dimer or thins the membrane via a monomer in the POPC/POG membrane. ${ }^{30}$ As shown by the computer simulations ${ }^{35}$ and other experimental results, ${ }^{22,31}$ the membrane thinning phenomenon at the initial state with higher $\mathrm{P} / \mathrm{L}$ ratios in addition to the formation of the isotropic phase was also detected. However, 100 days later, the membrane thinning disappeared along with the decrement of isotropic phase, which means that the isotropic phase of the lipid-peptide assembly is formed via membrane thinning by the adsorption of the peptides. The membrane thinning was generally identified by the decrement of anisotropic frequency span in ${ }^{2} \mathrm{H}$ SSNMR spectrum, indicated by the dashed lines.

Phase changes of POPC/cholesterol bilayers. In general, it has been known that cholesterol hardens a lipid membrane and therefore a higher concentration of the membrane-bound AMPs is required to disrupt the biomembrane. However, the experimental spectra shown in Fig. 5 did not show this trend when the $\mathrm{P} / \mathrm{L}$ ratio was over $1 / 80$. The membrane disruptions were similar to those in POPC membranes and even more disrupted at the $\mathrm{P} / \mathrm{L}$ ratios of $1 / 50$ and $1 / 20$. When the experimental spectra were simulated theoretically with the model of the mosaic spread of pore-containing bilayers, the lipid portions involved in the pore structures were $10 \%, 60 \%$, and $70 \%$ for the $1 / 80,1 / 50$, and $1 / 20$ samples, respectively. Considering the spectral signal-to-noise ratio, the simulations are well matched to the experimental spectra as shown in Fig. 5. The degrees of Gaussian spreads were also the same as those in the POPC cases with the standard deviation of $10-25^{\circ}$. At the high $\mathrm{P} / \mathrm{L}$ ratios $(1 / 50$ and $1 / 20)$, isotropic phases were shown at the center of the spectrum like those in the POPC samples, but the percentages were smaller than those found in the POPC samples. The isotropic phase does not show sharp peak unlike the POPC samples, which means that the iso- 


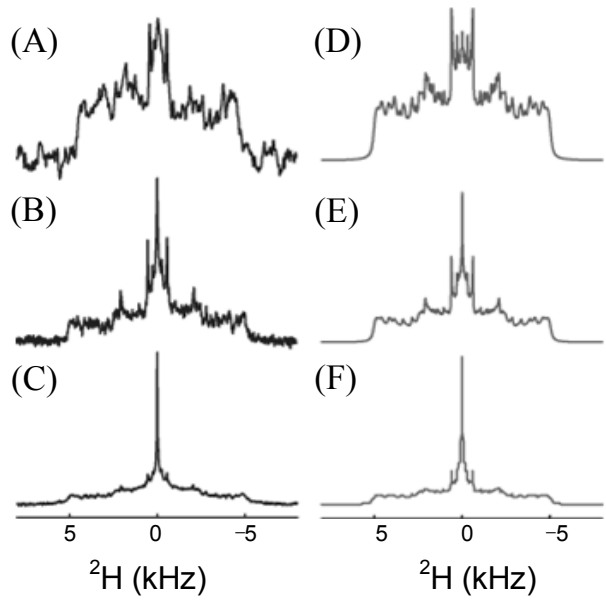

Figure 6. Experimental (A-C) and simulated (D-F) ${ }^{2} \mathrm{H}$ SSNMR spectra of POPC $d_{31} /$ cholesterol membrane disturbed by PG-1 with the P/L ratio of $1 / \overline{2} 0$. Spectra were measured (A) within a few days after sampling, (B) 100 days after sampling, and (C) 200 days after sampling. The spectra were simulated with (D) $70 \%$ of pores, $2 \%$ of an isotropic phase, and $28 \%$ of a Gaussian distribution of bilayers with a standard deviation of $25^{\circ}$, (E) $90 \%$ of pores, $5 \%$ of an isotropic phase, and $5 \%$ of a Gaussian distribution of bilayers with a standard deviation of $0^{\circ}$, (F) $80 \%$ of pores and $20 \%$ of an isotropic phase.

tropic phase was too large to rotate at a fast rate.

As measured in the POPC sample with the $\mathrm{P} / \mathrm{L}$ ratio of $1 / 20$, the time evolution of the mixture phase of the sample over 200 days was measured. As shown in Fig. 6, the spectra of the 1/20 sample had a greater percentage of the isotropic phase and a sharper peak at $0 \mathrm{ppm}$ than that observed in the $1 / 80$ and $1 / 50$ samples. The isotropic phase sharpened with longer incubation times, which means that the isotropic phase assembly gets smaller with an increase in the $\mathrm{P} / \mathrm{L}$ ratio in the peptide-lipid assembly.

The experimental spectra were simulated with the same model used in the POPC samples. The simulations matched well with the experimental spectra. The pore, isotropic, and bilayer portions and their standard deviation of the Gaussian distribution are given in the caption for Fig. 6. Contrary to the POPC case that induced a $100 \%$ isotropic phase, the cholesterol-containing POPC lipid membrane retained most pore structures over 200 days later, even though the percentage increased from $2 \%$ to $20 \%$.

\section{Conclusion}

The mixture phase of PG-1 and lipids was determined by measuring the ${ }^{2} \mathrm{H}$ SSNMR spectra of the sample and simulating the spectra with several reasonable phase models. The POPC lipid phase disturbed by an antimicrobial peptide, PG-1, changes slowly with time after hydration at $5{ }^{\circ} \mathrm{C}$. When the vacuum-dried peptide-lipid mixture was deposited on the thin cover-glass plate, pores were formed by the antimicrobial action of the peptide in the bilayers. However, the lipid bilayers containing the pores were not well aligned to the glass plate normal at the initial state and rather spread their bilayer normal directions by a Gaussian mosaic distribution. After over a hundred days, the bilayers with the pores were well aligned. An isotropic phase after a long incubation in a refrigerator was formed and coexisted with the bilayers containing the pores.

The peptide concentration effect on the bilayer disruption was apparent in the POPC and POPC/cholesterol bilayers, but was not clear in the POPC/POPG. As the $\mathrm{P} / \mathrm{L}$ ratio increases, an isotropic phase was formed both in the POPC and POPC/cholesterol bilayers. The critical value of the peptide concentration for the formation of an isotropic phase is higher in the POPC/cholesterol bilayer than in the POPC bilayer. The rotational diffusion rate of the isotropic phase decreased in the POPC/cholesterol bilayers, which may be caused by the larger size of the cholesterol/POPC/peptide assembly.

Acknowledgments. This paper has been supported by the 2009 Hannam University Research Fund.

\section{References}

1. Papagianni, M. Biotechnol. Adv. 2003, 21, 465.

2. Thomma, B. P.; Cammue, B. P.; Thevissen, K. Curr. Drug Targets: Infect. Disord. 2003, 3, 1.

3. Rozek, T.; Bowie, J. H.; Wallace, J. C.; Tyler, M. J. Rapid Commun. Mass Spectrom. 2000, 14, 2002.

4. Cole, A. M. Protein Pept. Lett. 2005, 12, 41.

5. Yount, N. Y.; Yeaman, M. R. Protein Pept. Lett. 2005, 12, 49.

6. Rozek, T.; Wegener, K. L.; Bowie, J. H.; Olver, I. N.; Carver, J. A.; Wallace, J. C.; Tyler, M. J. Eur. J. Biochem. 2000, 267, 5330.

7. Toke, O. Biopolymers 2005, 80, 717.

8. Kim, C.; Spano, J.; Park, E. K.; Wi, S. Biochim. Biophys. Acta 2009, 1788, 1482.

9. He, K.; Ludtke, S. J.; Worcester, D. L.; Huang, H. W. Biophys. J. 1996, 70, 2659.

10. Mani, R.; Waring, A. J.; Lehrer, R. I.; Hong, M. Biochim. Biophys. Acta, Biomembr. 2005, 1716, 11.

11. Bai, Y.; Liu, S.; Jiang, P.; Zhou, L.; Li, J.; Tang, C.; Verma, C.; Mu, Y.; Beuerman, R. W.; Pervushin, K. Biochemistry 2009, 48, 7229.

12. Toke, O. Biopolymers 2005, 80, 717.

13. Yeaman, M. R.; Yount, N. Y. Pharmacol. Rev. 2003, 55, 27.

14. Ludtke, S. J.; He, K.; Heller, W. T.; Harroun, T. A.; Yang, L.; Huang, H. W. Biochemistry 1996, 35, 13723.

15. Matsuzaki, K.; Murase, O.; Fujii, N.; Miyajima, K. Biochemistry 1996, 35, 11361.

16. Huang, H. W. Biochemistry 2000, 39, 8347.

17. Tang, Y. Q.; Yuan, J.; Osapay, G.; Osapay, K.; Tran, D.; Miller, C. J.; Ouellette, A. J.; Selsted, M. E. Science 1999, 286, 498.

18. Buffy, J. J.; McCormick, M. J.; Wi, S.; Waring, A.; Lehrer, R. I.; Hong, M. Biochemistry 2004, 43, 9800.

19. Meyer, C. E.; Reusser, F. Experientia 1967, 23, 85.

20. Matsuzaki, K. Biochim. Biophys. Acta, Rev. Biomembr. 1998, 1376, 391

21. Zasloff, M. Proc. Natl. Acad. Sci. U. S. A. 1987, 84, 5449.

22. Weiss, T. M.; Yang, L.; Ding, L.; Waring, A. J.; Lehrer, R. I.; Huang, H. W. Biochemistry 2002, 41, 10070.

23. Afonin, S.; Frey, A.; Bayerl, S.; Fischer, D.; Wadhwani, P.; Weinkauf, S.; Ulrich, A. S. ChemPhysChem 2006, 7, 2134.

24. Corzo, G.; Escoubas, P.; Villegas, E.; Barnham, K. J.; He, W.; Norton, R. S.; Nakajima, T. Biochem. J. 2001, 359, 35.

25. Nomura, K.; Corzo, G.; Nakajima, T.; Iwashita, T. Biophys. J. 2004, 87, 2497.

26. Prenner, E. J.; Lewis, R. N.; Jelokhani-Niaraki, M.; Hodges, R. S.; McElhaney, R. N. Biochim. Biophys. Acta 2001, 1510, 83.

27. Kokryakov, V. N.; Harwig, S. S.; Panyutich, E. A.; Shevchenko, A. A.; Aleshina, G. M.; Shamova, O. V.; Korneva, H. A.; Lehrer, R. I. FEBS Lett. 1993, 327, 231. 
28. Fahrner, R. L.; Dieckmann, T.; Harwig, S. S. L.; Lehrer, R. I.; Eisenberg, D.; Feigon, J. Chem. Biol. 1996, 3, 543.

29. Yang, L.; Weiss, T. M.; Lehrer, R. I.; Huang, H. W. Biophys. J. 2000, 79, 2002.

30. Mani, R.; Buffy, J. J.; Waring, A. J.; Lehrer, R. I.; Hong, M. Biochemistry 2004, 43, 13839.

31. Buffy, J. J.; Hong, T.; Yamaguchi, S.; Waring, A. J.; Lehrer, R. I.; Hong, M. Biophys. J. 2003, 85, 2363.

32. Buffy, J. J.; Waring, A. J.; Lehrer, R. I.; Hong, M. Biochemistry 2003, 42, 13725.

33. Yamaguchi, S.; Hong, T.; Waring, A.; Lehrer, R. I.; Hong, M. Biochemistry 2002, 41,9852.

34. Wi, S.; Kim, C. J. Phys. Chem. B 2008, 112, 11402.

35. Jang, H.; Ma, B.; Woolf Thomas, B.; Nussinov, R. Biophys. J. 2006, $91,2848$.
36. Buffy, J. J.; Waring, A. J.; Hong, M. J. Am. Chem. Soc. 2005 , $127,4477$.

37. Jang, H.; Ma, B.; Nussinov, R. BMC Struct. Biol. 2007, 7, 21.

38. Sayyed-Ahmad, A.; Kaznessis, Y. N. PLoS One 2009, 4, e4799.

39. Buffy, J. J.; McCormick, M. J.; Wi, S.; Waring, A.; Lehrer, R. I.; Hong, M. Biochemistry 2004, 43, 9800.

40. Strandberg, E.; Ulrich, A. S. Concepts Magn. Reson. A 2004, $23 A, 89$.

41. Hallock, K. J.; Lee, D.-K.; Ramamoorthy, A. Biophys. J. 2003, 84, 3052.

42. Dolainsky, C.; Unger, M.; Bloom, M.; Bayerl, T. M. Phys. Rev. E: Stat. Phys. Plasmas Fluids Relat. Interdisciplin. Top. 1995, 51, 4743.

43. Auger, M.; Smith, I. C.; Jarrell, H. C. Biophys. J. 1991, 59, 31.

44. Singer, S. J.; Nicolson, G. L. Science 1972, 175, 720. 